\title{
JBFnet - Low Dose CT Denoising by Trainable Joint Bilateral Filtering
}

\author{
Mayank Patwari ${ }^{1,2}[0000-0001-5197-5260]$, Ralf Gutjahr ${ }^{2}$, Rainer Raupach ${ }^{2}$, and \\ Andreas Maier ${ }^{1}$ \\ 1 Pattern Recognition Lab, Friedrich-Alexander Universitt Erlangen-Nrnberg (FAU), \\ Erlangen, Germany \\ 2 Siemens Healthcare GmbH, Forchheim, Germany \\ mayank.patwari@fau.de
}

\begin{abstract}
Deep neural networks have shown great success in low dose CT denoising. However, most of these deep neural networks have several hundred thousand trainable parameters. This, combined with the inherent non-linearity of the neural network, makes the deep neural network difficult to understand with low accountability. In this study we introduce JBFnet, a neural network for low dose CT denoising. The architecture of JBFnet implements iterative bilateral filtering. The filter functions of the Joint Bilateral Filter (JBF) are learned via shallow convolutional networks. The guidance image is estimated by a deep neural network. JBFnet is split into four filtering blocks, each of which performs Joint Bilateral Filtering. Each JBF block consists of 112 trainable parameters, making the noise removal process comprehendable. The Noise Map (NM) is added after filtering to preserve high level features. We train JBFnet with the data from the body scans of 10 patients, and test it on the AAPM low dose CT Grand Challenge dataset. We compare JBFnet with state-of-the-art deep learning networks. JBFnet outperforms CPCE3D, GAN and deep GFnet on the test dataset in terms of noise removal while preserving structures. We conduct several ablation studies to test the performance of our network architecture and training method. Our current setup achieves the best performance, while still maintaining behavioural accountability.
\end{abstract}

Keywords: Low Dose CT Denoising - Joint Bilateral Filtering · Precision Learning · Convolutional Neural Networks

\section{Background}

Reducing the radiation dose in clinical $\mathrm{CT}$ is extremely desirable to improve patient safety. However, lowering the radiation dose applied results in a higher amount of quantum noise in the reconstructed image, which can obscure crucial diagnostic information [17. Therefore, it is necessary to reduce the noise content while preserving all the structural information present in the image. So far, this is 


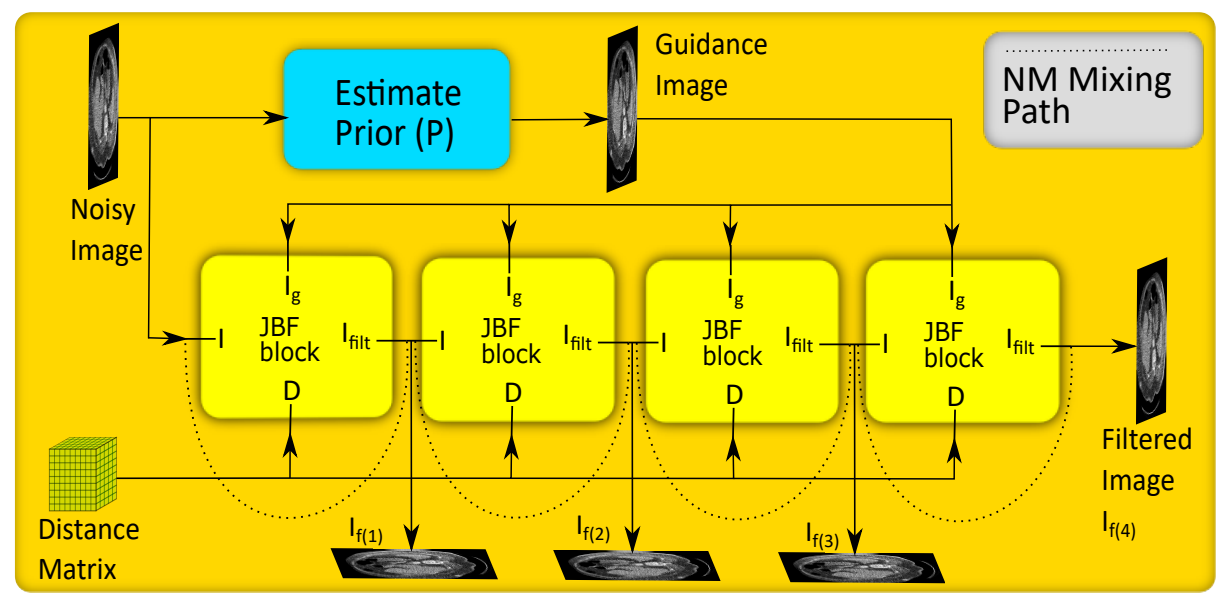

Fig. 1. The general architecture of JBFnet. There is a prior estimator and 4 JBF blocks for filtering. The NM mixing paths are shown in the diagram. The intermediate outputs of JBFnet are also shown.

achieved through non-linear filtering [13 14] and iterative reconstruction [356]. Most CT manufacturers use iterative reconstruction techniques in their latest scanners [118].

In the past decade, deep learning methods 4 have shown great success in a variety of image processing tasks such as classification, segmentation, and domain translation 7]. Deep learning methods have also been applied to clinical tasks, with varying degrees of success [16/22]. One such problem where great success has been achieved is low dose CT denoising [2|8|10|20|23|25|26]. Deep learning denoising methods outperform commercially used iterative reconstruction methods 19 .

Deep learning methods applied to the problem of low dose CT denoising are usually based on the concept of deep convolutional neural networks (CNN). Such CNNs have hundreds of thousands of trainable parameters. This makes the behaviour of such networks difficult to comprehend, which aggravates the difficulty of using such methods in regulated settings. This is why, despite their success, deep CNNs have only sparingly been applied in the clinic. It has been shown that including the knowledge of physical operations into a neural network can increase the performance of the neural network 11/12]21. This also helps to comprehend the behaviour of the network.

In this work, we introduce JBFnet, a CNN designed for low dose CT denoising. JBFnet implements iterative Joint Bilateral Filtering within its architecture. A deep CNN was used to generate the guidance image for the JBF. Shallow 2 layered CNNs were used to calculate the intensity range and spatial domain functions for the JBF. JBFnet has 4 sequential filtering blocks, to implement iterative filtering. Each block learns different range and domain filters. A portion of the NM is mixed back in after filtering, which helps preserve details. We 
demonstrate that JBFnet significantly outperforms denoising networks with a higher number of trainable parameters, while maintaining behavioural accountability.

\section{Methods}

\subsection{Trainable Joint Bilateral Filtering}

JBF takes into account the differences in both the pixel coordinates and the pixel intensities. To achieve this, the JBF has separate functions to estimate the appropriate filtering kernels in both the spatial domain and the intensity ranges. As opposed to bilateral filtering, the JBF uses a guidance image to estimate the intensity range kernel. The operation of the JBF is defined by the following equation:

$$
I_{f}(x)=\frac{\sum_{o \epsilon N(x)} I_{n}(o) B_{w}\left(I_{g}, x, o\right)}{\sum_{o \epsilon N(x)} B_{w}\left(I_{g}, x, o\right)} ; B_{w}\left(I_{g}, x, o\right)=G(x-o) F\left(I_{g}(x)-I_{g}(o)\right)
$$

where $I_{n}$ is the noisy image, $I_{f}$ is the filtered image, $x$ is the spatial coordinate, $N(x)$ is the neighborhood of $x$ taken into account for the filtering operation, $I_{g}$ is the guidance image, $F$ is the filtering function for estimating the range kernel, and $G$ is the filtering function for estimating the domain kernel.

We assume that $G$ and $F$ are defined by parameters $W_{g}$ and $W_{f}$ respectively. Similarly, we assume that the guidance image $I_{g}$ is estimated from the noisy image $I_{n}$ using a function $P$, whose parameters are defined by $W_{p}$. Therefore, we can rewrite Equation 1 as:

$$
I_{f}(x)=\frac{\sum_{o \epsilon N(x)} I_{n}(o) G\left(x-o ; W_{g}\right) F\left[P\left(I_{n}(x) ; W_{p}\right)-P\left(I_{n}(o) ; W_{p}\right) ; W_{f}\right]}{\sum_{o \in N(x)} G\left(x-o ; W_{g}\right) F\left[P\left(I_{n}(x) ; W_{p}\right)-P\left(I_{n}(o) ; W_{p}\right) ; W_{f}\right]}
$$

Since the values of $W_{f}$ act on the guidance image $I_{g}$ which is generated by $W_{p}$, we can rewrite $W_{f}$ as a function of $W_{p}$. The chain rule can be applied to get the gradient of $W_{p}$. The least squares problem to optimize our parameters is given by:

$$
\begin{aligned}
& L\left(W_{g}, W_{f}\left(W_{p}\right)\right)= \\
& \frac{1}{2}\left\|\frac{\sum_{o \epsilon N(x)} I_{n}(o) G\left(x-o ; W_{g}\right) F\left[P\left(I_{n}(x) ; W_{p}\right)-P\left(I_{n}(o) ; W_{p}\right) ; W_{f}\right]}{\sum_{o \epsilon N(x)} G\left(x-o ; W_{g}\right) F\left[P\left(I_{n}(x) ; W_{p}\right)-P\left(I_{n}(o) ; W_{p}\right) ; W_{f}\right]}-I_{f}(x)\right\|^{2}
\end{aligned}
$$

The gradients of $W_{g}$ and $W_{p}$ ( $\frac{\partial L}{\partial W_{g}}$ and $\frac{\partial L}{\partial W_{f}} \frac{\partial W_{f}}{\partial W_{p}}$ respectively) can be calculated using backpropogation and the parameters can by updated by standard gradient descent. 


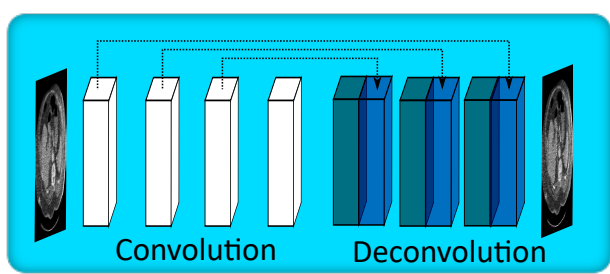

(a) Prior estimator block

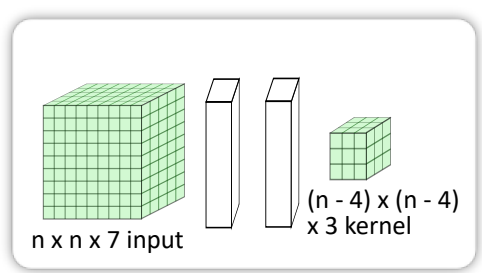

(b) Kernel estimator block

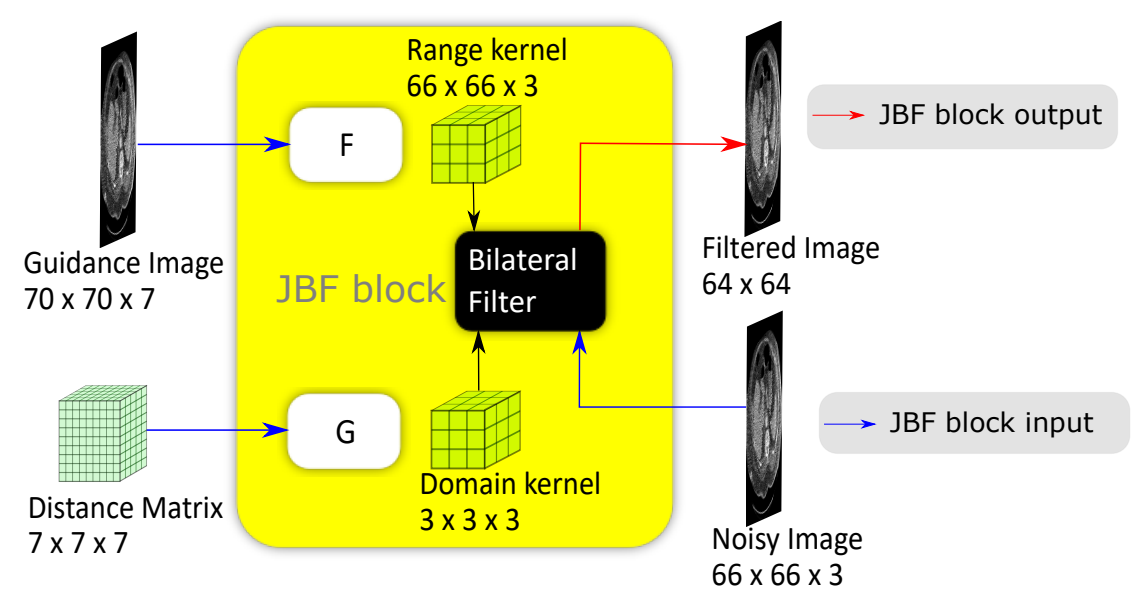

(c) Joint bilateral filtering block

Fig. 2. The architecture of (a) the prior estimator network $P$, (b) the kernel estimating functions $G$ and $F$ and (c) the functioning of the JBF block. Fig. 1 shows how to combine the components to create JBFnet.

\subsection{Network Architecture}

Prior Estimator Block The guidance image is estimated from the noisy image $I_{n}$ using a function $P$ with parameters $W_{p}$. We represent this function with a deep neural network inspired by CPCE3D 20. We have a hybrid 2D/3D network with 4 convolutional layers and 4 deconvolutional layers. Each layer has 32 filters. The filters in the convolutional layers are of size $3 \times 3 \times 3$. The filters in the deconvolutional layers are of size $3 \times 3$. Each layer is followed by a leaky ReLU activation function [9. $P$ takes an input of size $n \times n \times 15$, and returns an output of size $n \times n \times 7$. A structural diagram is present in Fig. 2(a).

JBF Block We introduce a novel denoising block called the JBF block. JBFnet performs filtering through the use of JBF blocks. Each JBF block contains two filtering functions, $G$ and $F$, with parameters $W_{g}$ and $W_{f}$ respectively. We represent each of these functions with a shallow convolutional network of 2 layers. Each layer has a single convolutional filter of size $3 \times 3 \times 3$. Each layer is fol- 


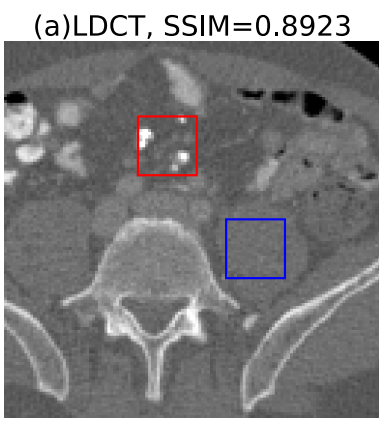

(d)GFnet, SSIM=0.9044

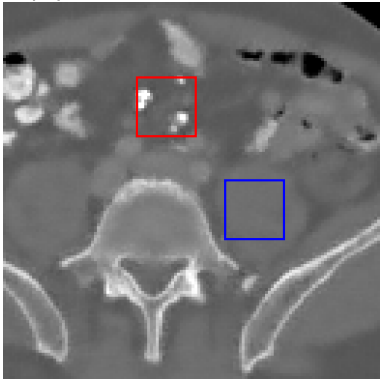

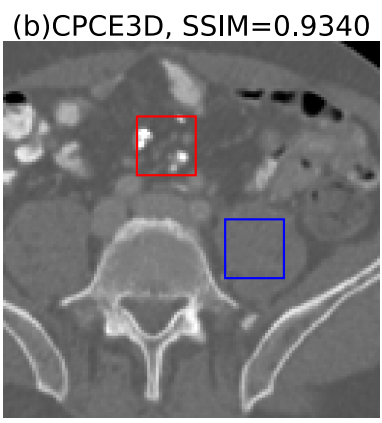

(e)JBFnet, $\mathrm{SSIM}=0.9391$

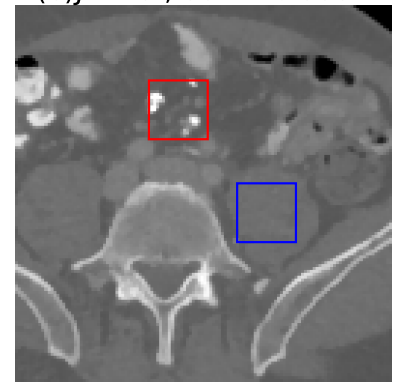

(c) GAN, SSIM $=0.8098$

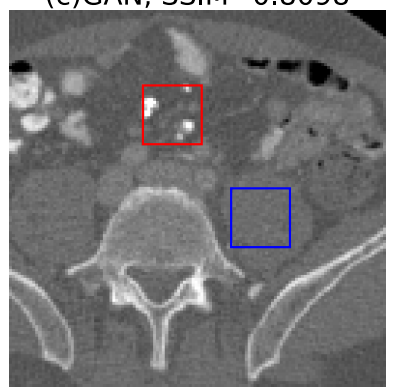

(f)SDCT, SSIM=1.0000

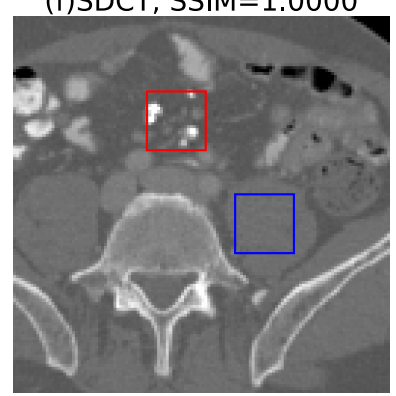

Fig. 3. We display an example of (a) low dose CT, denoised by (b) CPCE3D (c) GAN, (d) GFnet (e) JBFnet, and finally (f) the standard dose CT. The red square indicates some small low contrast features. The blue square indicates a homogenous noisy patch. SSIM scores are displayed in the captions. JBFnet achieves the best performance (SSIM $=0.9391)$. Images are displayed with a window of $[-800,1200]$.

lowed by a ReLU activation function [9. No padding is applied, shrinking an input of $n \times n \times 7$ to $(n-4) \times(n-4) \times 3$ (Fig. 2 (b)). The JBF block then executes a $3 \times 3 \times 3$ standard bilateral filtering operation with the outputs of $F$ and $G$. Each JBF block is followed by a ReLU activation function. JBFnet contains four consecutive JBF blocks (Fig. 1).

Mixing in the Noise Map After filtering with the JBF block, there is the possibilty that some important details may have been filtered out. To rectify this, we mixed an amount of the NM back into the filtered output (Fig. 1). The NM was estimated by subtracting the output of the JBF block from the input. We mix the NM by a weighted addition to the output of the JBF block. The weights are determined for each pixel by a $3 \times 3$ convolution of the NM.

Loss Functions We optimize the values of the parameters using two loss functions. We utilize the mean squared error loss and the edge filtration loss [19]. The two loss functions are given by the following equations:

$$
M S E L o s s\left(I_{1}, I_{2}\right)=\frac{1}{n}\left\|I_{1}-I_{2}\right\|^{2} ; \operatorname{EFLoss}\left(I_{1}, I_{2}\right)=\frac{1}{n}\left\|I_{1}^{\prime}-I_{2}^{\prime}\right\|^{2}
$$



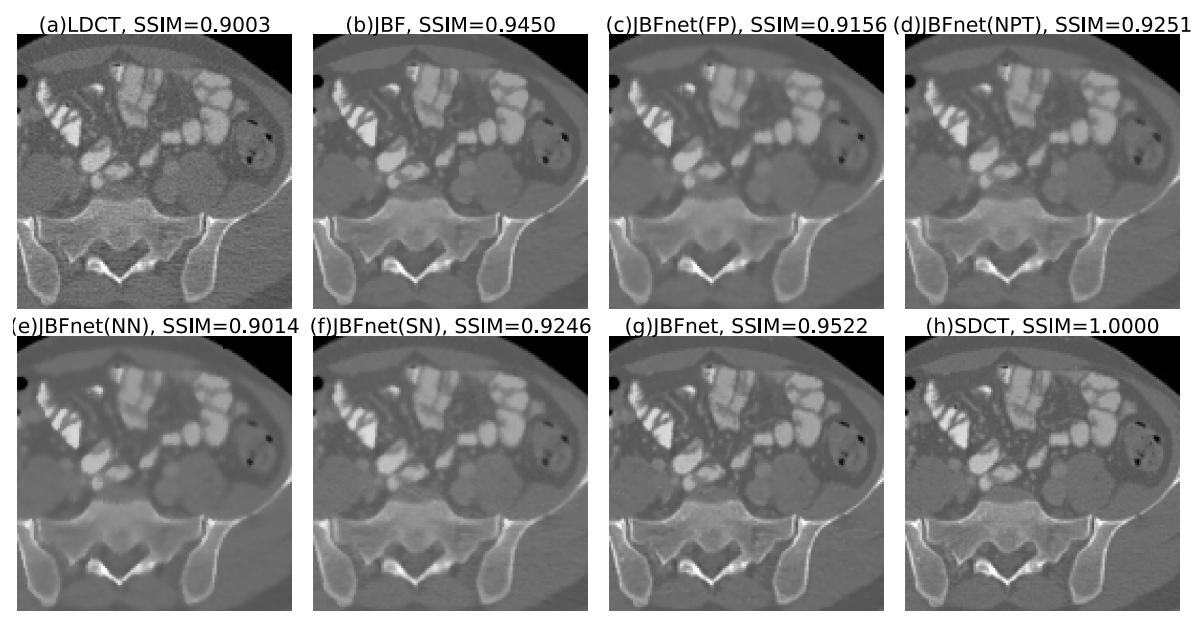

Fig. 4. We display a close-up example of (a) low dose CT, denoised by (b) JBF (c) JBFnet with frozen prior (FP), (d) JBFnet with no pre-training (NPT), (e) JBFnet with no NM (NN) added (f) JBFnet with single-weight NM (SN) added (g) JBFnet, and finally we display (e) the standard dose CT. SSIM scores are displayed in the captions. JBFnet achieves the best performance $($ SSIM $=0.9522)$. Images are displayed with a window of $[-800,1200]$.

$I_{1}^{\prime}$ and $I_{2}^{\prime}$ were computed using $3 \times 3 \times 3$ Sobel filters on $I_{1}$ and $I_{2}$ respectively. JBFnet outputs the estimated guidance image $I_{g}$, and the outputs of the four JBF blocks, $I_{f(1)}, I_{f(2)}, I_{f(3)}$ and $I_{f(4)}$ (Fig. 1). We indicate the reference image as $I$. Then, our overall loss function is:

$$
\begin{array}{r}
\lambda_{1}\left[M S E \operatorname{Loss}\left(I_{f(4)}, I\right)+0.1 * \operatorname{EFLoss}\left(I_{f(4)}, I\right)\right]+\lambda_{2}\left[M S E \operatorname{Loss}\left(I_{g}, I\right)\right]+ \\
\lambda_{3}\left[\sum_{i=1,2,3} \operatorname{MSELoss}\left(I_{f(i)}, I\right)+0.1 * \operatorname{EFLoss}\left(I_{f(i)}, I\right)\right]
\end{array}
$$

where the $\lambda$ values are the balancing weights of the loss terms in Equation 5 .

\subsection{Training and Implementation}

We trained JBFnet using the body scan data from 10 different patients. The scans were reconstructed using standard clinical doses, as well as $5 \%, 10 \%, 25 \%$ and $50 \%$ of the standard dose. The standard dose volumes are our reference volumes $I$. The reduced dose volumes are our noisy volumes $I_{n}$.

JBFnet was fed blocks of size $64 \times 64 \times 15$. The guidance image $I_{g}$ was estimated from the input, which was shrunk to $64 \times 64 \times 7$ by $P$. $I_{g}$ was then zero-padded to $70 \times 70 \times 7$, and then shrunk to a $66 \times 66 \times 3$ range kernel by $F$ (Fig. 2(c)). The distance matrix was constant across the whole image, and was pre-computed as a $7 \times 7 \times 7$ matrix, which was shrunk to a $3 \times 3 \times 3$ 
Table 1. Mean and standard deviations of the quality metric scores of our method and variants on the AAPM Grand Challenge dataset [15. The number of parameters in the networks are also included. Plain JBF application yields the highest PSNR $(46.79 \pm 0.8685)$, while JBFnet yields the highest SSIM $(0.9825 \pm 0.0025)$.

\begin{tabular}{||c||cc||}
\hline \multicolumn{1}{|c||}{} & PSNR & SSIM \\
\hline Low Dose CT & $43.12 \pm 1.021$ & $0.9636 \pm 0.0073$ \\
\hline CPCE3D [20] & $45.43 \pm 0.6914$ & $0.9817 \pm 0.0029$ \\
GAN [23] & $41.87 \pm 0.7079$ & $0.9398 \pm 0.0079$ \\
Deep GFnet [24] & $41.62 \pm 0.3856$ & $0.9709 \pm 0.0029$ \\
\hline JBF & $\mathbf{4 6 . 7 9} \pm \mathbf{0 . 8 6 8 5}$ & $0.9770 \pm 0.0046$ \\
JBFnet (Frozen Prior) & $42.86 \pm 0.4862$ & $0.9768 \pm 0.0027$ \\
JBFnet (No pre-training) & $42.75 \pm 0.5076$ & $0.9787 \pm 0.0027$ \\
JBFnet (No NM) & $42.64 \pm 0.5001$ & $0.9744 \pm 0.0029$ \\
JBFnet (Single-weight NM) & $42.07 \pm 0.5421$ & $0.9776 \pm 0.0029$ \\
\hline JBFnet & $44.76 \pm 0.6009$ & $\mathbf{0 . 9 8 2 5} \pm \mathbf{0 . 0 0 2 5}$ \\
\hline
\end{tabular}

kernel by $G$. The noisy input $I_{n}$ to the JBF blocks was a $64 \times 64 \times 3$ block, which contained the central 3 slices of the input block. $I_{n}$ was zero-padded to 66 $\times 66 \times 3$, and then shrunk to a $64 \times 64$ output by the bilateral filter (Fig. 2(c)). The NM was added in with learned pixelwise weights. After the JBF block, the output filtered image was padded by the neighbouring slices in both directions, restoring the input size to $64 \times 64 \times 3$ for future JBF blocks. This padding was not performed after the last JBF block, resulting in an overall output size of 64 $\times 64$.

Training was performed for 30 epochs over the whole dataset. 32 image slabs were presented per batch. For the first ten epochs, only $W_{p}$ was updated $\left(\lambda_{1}=\right.$ $\left.0, \lambda_{2}=1, \lambda_{3}=0\right)$. This was to ensure a good quality guidance image for the JBF blocks. From epochs $10-30$, all the weights were updated $\left(\lambda_{1}=1, \lambda_{2}=\right.$ $\left.0.1, \lambda_{3}=0.1\right)$. JBFnet was implemented in PyTorch on a PC with an Intel Xeon E5-2640 v4 CPU and an NVIDIA Titan Xp GPU.

\section{$3 \quad$ Experimental Results}

\subsection{Test Data and Evaluation Metrics}

We tested our denoising method on the AAPM Low Dose CT Grand Challenge dataset [15. The dataset consisted of 10 body CT scans, each reconstructed at standard doses as used in the clinic and at $25 \%$ of the standard dose. The slices were of $1 \mathrm{~mm}$ thickness. We aimed to map the reduced dose images onto the standard dose images. The full dose images were treated as reference images. Inference was performed using overlapping $256 \times 256 \times 15$ blocks extracted from the test data.

We used the PSNR and the SSIM to measure the performance of JBFnet. Since structural information preservation is more important in medical imaging, the SSIM is a far more important metric of CT image quality than the 
PSNR. Due to the small number of patients, we used the Wilcoxon signed test to measure statistical significance. A p-value of 0.05 was used as the threshold for determining statistical significance.

\subsection{Comparison to State-of-the-Art Methods}

We compare the denoising performance of JBFnet to other denoising methods that use deep learning. We compare JBFnet against CPCE3D [20], GAN [23], and deep GFnet 24] (Fig. 3). All networks were trained over 30 epochs on the same dataset. JBFnet achieves significantly higher scores in both PSNR and SSIM compared to GAN and deep GFnet $(\mathrm{w}=0.0, \mathrm{p}=0.005)$. CPCE3D achieves a significantly higher PSNR than JBFnet $(\mathrm{w}=0.0, \mathrm{p}=0.005)$, but a significantly lower SSIM ( $\mathrm{w}=0.0, \mathrm{p}=0.005)$ (Table 1). Additionally, the JBF block consists of only 112 parameters, compared to the guided filtering block 24] which contains 1,555 parameters.

\subsection{Ablation Study}

Training the Filtering Functions Usually, the filter functions $F$ and $G$ of the bilateral filter are assumed to be Gaussian functions. We check if representing these functions with convolutions improves the denoising performance of our network. Training the filtering functions reduces our PSNR $(\mathrm{w}=0.0, \mathrm{p}=0.005)$ but improves our SSIM ( $\mathrm{w}=0.0, \mathrm{p}=0.005)$ (Table 1 and Fig. 4 ).

Pre-training the Prior Estimator In our current training setup, we exclusively train the prior estimator $P$ for 10 epochs, to ensure a good quality prior image. We check if avoiding this pre-training, or freezing the value of $P$ after training improves the performance of our network. Both freezing $P$ and not doing any pre-training reduce the PSNR and $\operatorname{SSIM~}(\mathrm{w}=0.0, \mathrm{p}=0.005)$ (Table 1 and Fig. 4).

Pixelwise Mixing of the NM Currently, we estimate the amount of the NM to be mixed back in by generating pixelwise coeffecients from a single $3 \times 3$ convolution of the NM. We check if not adding in the NM, or adding in the NM with a fixed weight improves the denoising performance of the network. Not mixing in the NM reduces our PSNR and SSIM significantly $(\mathrm{w}=0.0, \mathrm{p}$ $=0.005)$. Mixing in the NM with a fixed weight reduces both PSNR and SSIM even futher $(\mathrm{w}=0.0, \mathrm{p}=0.005)$ (Table 1 and Fig. 4 ).

\section{Conclusion}

In this study, we introduced JBFnet, a neural network which implements Joint Bilateral Filtering with learnable parameters. JBFnet significantly improves the denoising performance in low dose CT compared to standard Joint Bilateral 
Filtering. JBFnet also outperforms state-of-the-art deep denoising networks in terms of structural preservation. Furthermore, most of the parameters in JBFnet are present in the prior estimator. The actual filtering operations are divided into various JBF blocks, each of which has only 112 trainable parameters. This allows JBFnet to denoise while still maintaining physical interpretability.

\section{References}

1. Angel, E.: AIDR 3D Iterative Reconstruction : Integrated, Automated and Adaptive Dose Reduction (2012), https://us.medical.canon/download/ aidr-3d-wp-aidr-3d

2. Fan, F., Shan, H., Kalra, M.K., Singh, R., Qian, G., Getzin, M., Teng, Y., Hahn, J., Wang, G.: Quadratic Autoencoder (Q-AE) for Lowdose CT Denoising. IEEE Transactions on Medical Imaging (2019). https://doi.org/10.1109/tmi.2019.2963248

3. Gilbert, P.: Iterative methods for the three-dimensional reconstruction of an object from projections. Journal of Theoretical Biology 36(1), 105-117 (1972). https://doi.org/10.1016/0022-5193(72)90180-4

4. Goodfellow, I.J., Bengio, Y., Courville, A.: Deep Learning (2014). https://doi.org/10.1016/B978-0-12-801775-3.00001-9

5. Hounsfield, G.N.: Computerized transverse axial scanning (tomography). British Journal of Radiology 46(552), 1016-1022 (1973). https://doi.org/10.1259/00071285-46-552-1016

6. Kaczmarz, S.: Angenäherte Auflösung von Systemen linearer Gleichungen. Bulletin International de l'Académie Polonaise des Sciences et des Lettres. Classe des Sciences Mathématiques et Naturelles. Série A, Sciences Mathématiques 35, 355-357 (1937)

7. Lecun, Y., Bengio, Y., Hinton, G.: Deep learning. Nature 521(7553), 436-444 (2015). https://doi.org/10.1038/nature14539

8. Li, M., Hsu, W., Xie, X., Cong, J., Gao, W.: SACNN : Self-Attention Convolutional Neural Network for Low-Dose CT Denoising with Self-supervised Perceptual Loss Network. IEEE Transactions on Medical Imaging (2020). https://doi.org/10.1109/TMI.2020.2968472

9. Maas, A.L., Hannun, A.Y., Ng, A.Y.: Rectifier nonlinearities improve neural network acoustic models. In: International Conference on Machine Learning. vol. 30, p. 6 (2013)

10. Maier, A., Fahrig, R.: GPU Denoising for Computed Tomography. In: Xun, J., Jiang, S. (eds.) Graphics Processing Unit-Based High Performance Computing in Radiation Therapy, pp. 113-128. CRC Press, Boca Raton, Florida, USA, 1 edn. (2015)

11. Maier, A., Schebesch, F., Syben, C., Wurfl, T., Steidl, S., Choi, J.H., Fahrig, R.: Precision Learning: Towards Use of Known Operators in Neural Networks. In: Proceedings of the International Conference on Pattern Recognition. pp. 183-188. No. 2 (2018). https://doi.org/10.1109/ICPR.2018.8545553

12. Maier, A.K., Syben, C., Stimpel, B., Würfl, T., Hoffmann, M., Schebesch, F., Fu, W., Mill, L., Kling, L., Christiansen, S.: Learning with known operators reduces maximum error bounds. Nature Machine Intelligence 1(8), 373-380 (2019). https://doi.org/10.1038/s42256-019-0077-5 
13. Manduca, A., Yu, L., Trzasko, J.D., Khaylova, N., Kofler, J.M., McCollough, C.M., Fletcher, J.G.: Projection space denoising with bilateral filtering and CT noise modeling for dose reduction in CT. Medical Physics 36(11), 4911-4919 (2009). https://doi.org/10.1118/1.3232004

14. Manhart, M., Fahrig, R., Hornegger, J., Doerfler, A., Maier, A.: Guided Noise Reduction for Spectral CT with Energy-Selective Photon Counting Detectors. In: Proceedings of the Third CT Meeting. pp. 91-94. No. 1 (2014)

15. Mccollough, C.H.: Low Dose CT Grand Challenge (2016)

16. Nie, D., Trullo, R., Lian, J., Petitjean, C., Ruan, S., Wang, Q., Shen, D.: Medical Image Synthesis with Context-Aware Generative Adversarial Networks. In: Medical Image Computing and Computer Assisted Intervention. vol. 3, pp. 417-425 (2017). https://doi.org/10.1007/978-3-319-66179-7

17. Oppelt, A.: Noise in Computed Tomography. In: Aktiengesselschaft, S. (ed.) Imaging Systems for Medical Diagnostics, chap. 13.1.4.2, p. 996. Publicis Corporate Publishing, 2 edn. (2005). https://doi.org/10.1145/2505515.2507827

18. Ramirez-Giraldo, J.C., Grant, K.L., Raupach, R.: ADMIRE : Advanced Modeled Iterative Reconstruction (2015), https://www.siemens-healthineers.com/ computed-tomography/technologies-innovations/admire

19. Shan, H., Padole, A., Homayounieh, F., Kruger, U., Khera, R.D., Nitiwarangkul, C., Kalra, M.K., Wang, G.: Competitive performance of a modularized deep neural network compared to commercial algorithms for low-dose CT image reconstruction. Nature Machine Intelligence 1(6), 269-276 (2019). https://doi.org/10.1038/s42256019-0057-9

20. Shan, H., Zhang, Y., Yang, Q., Kruger, U., Kalra, M.K., Sun, L., Cong, W., Wang, G.: 3D Convolutional Encoder-Decoder Network for Low-Dose CT via Transfer Learning from a 2D Trained Network. IEEE Transactions on Medical Imaging 37(6), 1522 - 1534 (2018). https://doi.org/10.4172/2157-7633.1000305

21. Syben, C., Stimpel, B., Breininger, K., Würfl, T., Fahrig, R., Dörfler, A., Maier, A.: Precision Learning: Reconstruction Filter Kernel Discretization. In: Proceedings of the 5th International Conference on Image Formation in X-ray Computed Tomography. pp. 386-390. Salt Lake City (2017)

22. Wolterink, J.M., Dinkla, A.M., Savenije, Mark, H., Seevinck, P.R., van den Berg, C.A., Isgum, I.: Deep MR to CT Synthesis Using Unpaired Data. In: Workshop on Simulation and Synthesis in Medical Imaging. pp. 14-23 (2017). https://doi.org/10.1007/978-3-319-68127-6

23. Wolterink, J.M., Leiner, T., Viergever, M.A., Isgum, I.: Generative Adversarial Networks for Noise Reduction in Low-Dose CT. IEEE Trancations on Medical Imaging 36(12), 2536-2545 (2017). https://doi.org/10.1109/TMI.2017.2708987

24. Wu, H., Zheng, S., Zhang, J., Huang, K.: Fast End-to-End Trainable Guided Filter. In: Computer Vision and Pattern Recognition. pp. 1838-1847 (2018)

25. Yang, Q., Yan, P., Zhang, Y., Yu, H., Shi, Y., Mou, X., Kalra, M.K., Zhang, Y., Sun, L., Wang, G.: Low-Dose CT Image Denoising Using a Generative Adversarial Network With Wasserstein Distance and Perceptual Loss. IEEE Transactions on Medical Imaging 37(6), 1348-1357 (2018). https://doi.org/10.1109/TMI.2018.2827462

26. Zhang, Y., Wang, G., Zhang, W., Li, K., Chen, H., Liao, P., Zhou, J.: Low-dose CT via convolutional neural network. Biomedical Optics Express 8(2), 679 - 694 (2017). https://doi.org/10.1364/boe.8.000679 